\title{
Time to Evolve Terminology from "Debulking" to Cytoreductive Surgery (CRS) in Ovarian Cancer
}

\author{
Donal J. Brennan, MB, FRCPI, PhD ${ }^{1}$, and Brendan J. Moran, MB BCh, FRCSI, MCh, Doc Med ${ }^{2}$ \\ ${ }^{1}$ UCD Gynaecological Oncology Group, Catherine McAuley Research Centre, UCD School of Medicine, Mater \\ Misericordiae University Hospital, Dublin 7, Ireland; ${ }^{2}$ Peritoneal Malignancy Institute, Basingstoke and North Hampshire \\ Hospital, Basingstoke, UK
}

\author{
"where do those old fellows get all the words they \\ have"
}

Molly Bloom re "Dr Collins for women's diseases", Ulysses by James Joyce

The importance of language in medicine is often overlooked. Similar to survival rates in epithelial ovarian cancer, which have remained relatively static over the last three decades, it is clear that the terminology used when describing surgical procedures for advanced epithelial ovarian cancer has not evolved from the rather non-descriptive term "debulking". While most authorities accept that surgery is pivotal and that complete macroscopic resection is now considered optimal standard of care, the terms "primary debulking surgery" and "interval debulking surgery" are poor descriptions for what are complex and intricate procedures.

The inverse relationship between residual disease following "debulking surgery" and survival in ovarian cancer has been confirmed in multiple cohort studies, randomized control trials and meta-analyses since first described by the American gynaecologic oncologist C. Tom Griffiths in 1975. ${ }^{1}$ Over the last 45 years, the volume of residual disease following surgery for ovarian cancer has been defined as the diameter of the largest residual tumour mass. Initially described as residual disease $<2 \mathrm{~cm}$, the definition of

(C) Society of Surgical Oncology 2021

First Received: 4 June 2021

Accepted: 7 July 2021;

Published Online: 24 July 2021

D. J. Brennan, MB, FRCPI, PhD

e-mail: donal.brennan@ucd.ie "optimal debulking" has changed over time and is now considered to be "complete gross resection", which is described using a variety of terms including "no macroscopic disease" or "zero-residual". Despite the lack of standardized descriptive terms, outcomes associated with complete surgical excision are clear. A meta-analysis of 81 studies by Bristow and colleagues reported a $5.5 \%$ increase in median survival for each $10 \%$ increase in the proportion of women who had complete tumour resection. ${ }^{2}$

The quantification of both per-operative disease burden and post-operative residual disease in advanced ovarian cancer is subject to significant intra- and inter-observer variability, particularly with low-volume miliary peritoneal disease. Although historical evidence suggests that the number and extent of small lesions may have a significant impact on survival, ${ }^{3}$ current practice is that women with residual volume $<1 \mathrm{~cm}$ are all included in one category. This is particularly important when one takes account of the seminal papers from Eisenkop et al. ${ }^{4}$ and Bristow et al. ${ }^{5}$ which demonstrated survival benefits after meticulous resection of low-volume disease. Although these studies are over 20 years old, there has been little, or no, advancement in classification, and reproducible reporting, of residual disease since then.

In the parallel world of gastro-intestinal peritoneal malignancy, the combination strategy of what is now termed cytoreductive surgery (CRS) and hyperthermic intraperitoneal chemotherapy (HIPEC) has become synonymous with state-of-the-art treatment for peritoneal malignancy of gastro-intestinal origin. CRS and HIPEC is now accepted as optimal treatment of appendiceal pseudomyxoma peritonei $^{6}$ and malignant abdominal mesothelioma $^{7}$ and in selected patients with resectable colorectal peritoneal metastases. ${ }^{8}$ Although 
much of the emphasis within the medical literature, and in general discourse, has focused on the potential toxicity and questionable benefit of HIPEC, many overlook that there are two components to this strategy, namely the surgical CRS and the chemotherapeutic HIPEC, both with a different focus and mode of action. In the authors' opinion, optimal CRS is the key to almost all surgical interventions for peritoneal metastases with an adjunctive role for precise, targeted HIPEC which theoretically may have a role in control, or elimination, of small-volume metastases.

Two large phase 3 trials have sparked worldwide debate regarding the role of HIPEC in common peritoneal malignancies, namely the PRODIGE 7 trial for colorectal peritoneal metastases ${ }^{9}$ and the OVHIPEC trial for epithelial ovarian cancer. ${ }^{10}$ PRODIGE 7 has been described as the death knell for HIPEC in colorectal cancer; however, as median overall survival in both groups was 42 months, it suggests that optimal CRS works and that the specific HIPEC regimen used (oxaliplatin at $360-460 \mathrm{mg} / \mathrm{m}^{2}$ for 30 min) contributed little, if at all, but increased morbidity. Despite demonstrating a clear improvement in overall survival, with no additional major morbidity, the OVHIPEC study has been the subject of significant criticism. The OVHIPEC trial did take a long time to accrue, and peroperative randomization could contribute a surgical bias whereby more complete CRS might be performed if the surgeon knows that the patient is to receive HIPEC. Despite these criticisms, the most recent National Comprehensive Cancer Network (NCNN) guidelines have included HIPEC as an option at interval CRS, ${ }^{11}$ and Ghirardi et al. recently suggested that the OVHIPEC protocol can be applied in $35 \%$ of women undergoing interval CRS in a "real-life" setting, with no additional morbidity or delay in commencement of adjuvant therapy. ${ }^{12}$ The one central theme to both PRODIGE 7 and OVHIPEC, in our opinion, is that HIPEC may indeed be a "carrier solution" for optimal CRS.

Two key elements have emerged in CRS for gastrointestinal tract (GIT) peritoneal malignancy, namely the extent of disease at laparotomy, as measured by the peritoneal cancer index (PCI) ${ }^{13}$ and the completeness of cytoreduction (CC), as documented by the CC scoring system. ${ }^{13}$ The PCI is a composite of 13 abdominal regions (4 are small bowel) with size of tumour nodules scoring $0-3$. The PCI score thus ranges from 0 to 39. Even with four regions categorizing small bowel disease, in reality, extensive small bowel involvement will often be the factor precluding complete cytoreduction, irrespective of the origin of the primary tumour.

The CC scoring system quantifies the extent of the resection, and unsurprisingly, for almost all peritoneal malignancies, complete cytoreduction (removal of all macroscopic tumour) is associated with improved survival. ${ }^{14,15}$ The CC scoring system categorizes residual disease into $\mathrm{CC} 0$, no residual disease; $\mathrm{CC} 1$, residual disease nodules up to $2.5 \mathrm{~mm}$ in size; $\mathrm{CC} 2$, residual disease 2.5-25 mm; and CC 3 , residual tumour nodules greater than $25 \mathrm{~mm} .{ }^{13}$ Compared with residual disease measurement employed in ovarian cancer, the CC score is more likely to give a clear, reproducible description of residual disease.

The importance of quantification of disease burden by PCI and completeness of surgical excision CC scoring has helped to advance knowledge, and experience, of GIT malignancies, and aids appropriate selection, design of trials and intra-operative decision-making, and optimizes outcome. Furthermore, both systems complement each other and allow for clear comparison between patients, and units, and have facilitated important large international collaborative studies. ${ }^{7,14}$ The CC system incorporates the fact that hyperthermic intraperitoneal chemotherapy (HIPEC) may help address microscopic residual disease and penetrates tumour to a depth of $2.5-3.0 \mathrm{~mm}$ such that patients who have cytoreduction to $\mathrm{CC} 0$, or $\mathrm{CC} 1$, are theoretically amenable to a therapeutic benefit, provided that the drug is appropriately cytotoxic. Thus, for pseudomyxoma peritonei, $\mathrm{CC} 0$ and $\mathrm{CC} 1$ are categorized as complete macroscopic tumour removal with equivalent disease-free and overall survival. ${ }^{14}$

The implementation of the PCI and CC scoring systems in epithelial ovarian cancer would lead to greater standardization and reproducibility of surgical outcomes. The volume and location of retroperitoneal and extra-abdominal (inguinal, cardiophrenic or supraclavicular) nodal disease burden and residual disease should also be clearly documented given the well-described survival benefits associated with resection of enlarged lymph nodes in women who have a CC0 peritoneal resection. ${ }^{16}$ There are clearly major international variations in the standard of ovarian cancer surgery; however, these are not solely linked to resources alone. In many ways, gynaecological oncologists and surgical oncologists are simply not speaking the same language. Collaboration and interdisciplinary surgical teams working together can optimize CRS in epithelial ovarian cancer. ${ }^{17}$ Common terminology is helpful as all roads lead to optimal surgery.

On this background, HIPEC used in appropriate, evidence-based indications may add more than its current status as a carrier solution for optimal CRS. The time has come to remove "debulking" from the ovarian cancer medical literature and change the terminology to cytoreductive surgery. CRS should be offered to suitable patients with peritoneal malignancy. The extent of disease at laparotomy should be documented, and residual disease after optimal surgery should be quantified in a standardized and reproducible manner. The use, and meaning, of language in medicine is often underestimated, as outlined by 
Joyce almost one century ago. The time for change is now, and the methods are already in use in many fields of peritoneal malignancy.

\section{REFERENCES}

1. Griffiths CT. Surgical resection of tumor bulk in the primary treatment of ovarian carcinoma. Natl Cancer Inst Monogr. 1975;42:101-4.

2. Bristow RE, Tomacruz RS, Armstrong DK, et al. Survival effect of maximal cytoreductive surgery for advanced ovarian carcinoma during the platinum era: a meta-analysis. J Clin Oncol. 2002;20(5):1248-59.

3. Farias-Eisner R, Teng F, Oliveira M, et al. The influence of tumor grade, distribution, and extent of carcinomatosis in minimal residual stage III epithelial ovarian cancer after optimal primary cytoreductive surgery. Gynecol Oncol. 1994;55(1):108-10.

4. Eisenkop SM, Friedman RL, Wang HJ. Complete cytoreductive surgery is feasible and maximizes survival in patients with advanced epithelial ovarian cancer: a prospective study. Gynecol Oncol. 1998;69(2):103-8.

5. Bristow RE, Montz FJ. Complete surgical cytoreduction of advanced ovarian carcinoma using the argon beam coagulator. Gynecol Oncol. 2001;83(1):39-48.

6. Govaerts K, Lurvink RJ, De Hingh IHJT, et al. Appendiceal tumours and pseudomyxoma peritonei: literature review with PSOGI/EURACAN clinical practice guidelines for diagnosis and treatment. Eur J Surg Oncol. 2021;47(1):11-35.

7. Yan TD, Deraco M, Baratti D, et al. Cytoreductive surgery and hyperthermic intraperitoneal chemotherapy for malignant peritoneal mesothelioma: multi-institutional experience. $J$ Clin Oncol. 2009;27(36):6237-42.

8. Moran BJ, Cecil TD. Treatment of surgically resectable colorectal peritoneal metastases. Br J Surg. 2014;101(2):5-7.

9. Quénet F, Elias D, Roca L, et al. Cytoreductive surgery plus hyperthermic intraperitoneal chemotherapy versus cytoreductive surgery alone for colorectal peritoneal metastases (PRODIGE 7): a multicentre, randomised, open-label, phase 3 trial. Lancet Oncol. 2021;22(2):256-66.

10. van Driel WJ, Koole SN, Sikorska K, et al. Hyperthermic intraperitoneal chemotherapy in ovarian cancer. $N$ Engl J Med. 2018;378(3):230-40.

11. Armstrong DK, Alvarez RD, Bakkum-Gamez JN, et al. NCCN guidelines insights: ovarian cancer, version 1.2019. J Natl Compr Cancer Netw. 2019;17(8):896-909.

12. Ghirardi V, Ronsini C, Trozzi R, et al. Hyperthermic intraperitoneal chemotherapy in interval debulking surgery for advanced epithelial ovarian cancer: a single-center, real-life experience. Cancer. 2020;126(24):5256-62.

13. Jacquet $\mathrm{P}$, Sugarbaker PH. Clinical research methodologies in diagnosis and staging of patients with peritoneal carcinomatosis. Cancer Treat Res. 1996;82:359-74.

14. Chua TC, Moran BJ, Sugarbaker PH, et al. Early- and long-term outcome data of patients with pseudomyxoma peritonei from appendiceal origin treated by a strategy of cytoreductive surgery and hyperthermic intraperitoneal chemotherapy. J Clin Oncol. 2012;30(20):2449-56.

15. Ansari N, Chandrakumaran K, Dayal S, et al. Cytoreductive surgery and hyperthermic intraperitoneal chemotherapy in 1000 patients with perforated appendiceal epithelial tumours. Eur $J$ Surg Oncol. 2016;42(7):1035-41.

16. du Bois A, Reuss A, Harter P, et al. Potential role of lymphadenectomy in advanced ovarian cancer: a combined exploratory analysis of three prospectively randomized phase III multicenter trials. J Clin Oncol. 2010;28(10):1733-9.

17. Mulligan KM, Glennon K, Donohoe F, et al. Multidisciplinary surgical approach to increase complete cytoreduction rates for advanced ovarian cancer in a tertiary gynecologic oncology center. Ann Surg Oncol. 2021;28(8):4553-60. https://doi.org/10. 1245/s10434-020-09494-3.

Publisher's Note Springer Nature remains neutral with regard to jurisdictional claims in published maps and institutional affiliations. 